\title{
Square-wave thin-film voltammetry: influence of uncompensated resistance and charge transfer kinetics
}

\author{
Valentin Mirčeski ${ }^{\mathrm{a}, *}$, Rubin Gulaboski ${ }^{\mathrm{b}}$, Fritz Scholz ${ }^{\mathrm{b}}$ \\ a Institute of Chemistry, Faculty of Natural Sciences and Mathematics, "Sv. Kiril i Metodij” University, P.O. Box 162, 1000 Skopje, Macedonia \\ ${ }^{\mathrm{b}}$ Institut für Chemie und Biochemie, Ernst-Moritz-Arndt-Universität Greifswald, Soldmannstraße 23, D-17489 Greifswald, Germany
}

Received 22 July 2003; received in revised form 12 November 2003; accepted 13 November 2003

\begin{abstract}
An electrode reaction occurring in a thin-film having a low electrical conductivity was considered under conditions of squarewave voltammetry (SWV). The ohmic polarization of the system owing to the low conductivity of the film is represented by the complex resistance parameter defined as $\rho=R_{\Omega}\left(n^{2} F^{2} / R T\right) S c_{\mathrm{Ox}}^{*} \sqrt{D f}$, where $R_{\Omega}$ is the resistance of the film, $S$ is the electrode surface area, $f$ is the SW frequency and the other symbols have their usual meanings. The influence of the thickness of the film is given by the thickness parameter $\Lambda=L \sqrt{f / D}$, where $L$ is the thickness of the layer, and $D$ is the diffusion coefficient. For a thin film $(\Lambda \leqslant 0.949)$ the dimensionless net-peak current of a reversible electrode reaction depends parabolically on the resistance parameter, exhibiting a well-developed maximum. A detailed study of this phenomenon revealed that it resembles the charge transfer kinetics effect as well as the property known as a "quasireversible maximum" that is typical for an adsorption complicated electrode reaction. The theoretically predicted properties of the SW response are illustrated by experiments with iodine, decamethylferrocene, and azobenzene at the three-phase electrode system. This methodology [Electrochem. Commun. 2 (2000) 112] consists of a single droplet of an organic water immiscible solvent containing an electroactive probe that is attached to the surface of a graphite electrode and immersed in an aqueous electrolyte solution. A good agreement between theory and experiment indicates that the SW voltammetric response of the three-phase electrode system exhibits properties of an electrode reaction occurring under limiting diffusion conditions.
\end{abstract}

(c) 2003 Elsevier B.V. All rights reserved.

Keywords: Thin-film electrode; Three-phase electrode; Square-wave voltammetry

\section{Introduction}

The importance of the charge transfer across the liquid|liquid $(\mathrm{L} \mid \mathrm{L})$ interface for living systems is well recognized and intensively studied over the last several decades [1,2]. Besides the standard experimental arrangement for studying the ion transfer at the interface of immiscible electrolyte solutions (ITIES) based on a four electrode configuration [3-7], in recent years a few attempts have been made to utilize a three electrode arrangement to assess both the ion and the electron transfer processes across the $\mathrm{L} \mid \mathrm{L}$ interface. Anson and co-workers [8-13] used a solid graphite electrode, the

\footnotetext{
${ }^{*}$ Corresponding author. Tel.: +389-2-3117055; fax: +389-2-3226865.

E-mail address: valentin@iunona.pmf.ukim.edu.mk (V. Mirčeski).
}

surface of which is completely covered with a thin film of an organic liquid containing an electroactive probe and immersed in an aqueous electrolyte (see Scheme 1). The electron transfer at the carbon|organic liquid interface is accompanied by either ion or both ion and electron transfer across the organic liquid |aqueous solution interface. The method possesses a number of advantages and it is particularly useful for studying the electron transfer across the $\mathrm{L} \mid \mathrm{L}$ interface utilizing a simple experimental arrangement [10]. However, this methodology is sensitive to the conductivity of the organic film, which can be provided by partition of the electrolyte, initially present only in the aqueous phase, or alternatively by adding an organic electrolyte to the organic phase. When the conductivity is provided by partition between the aqueous electrolyte and the film, 


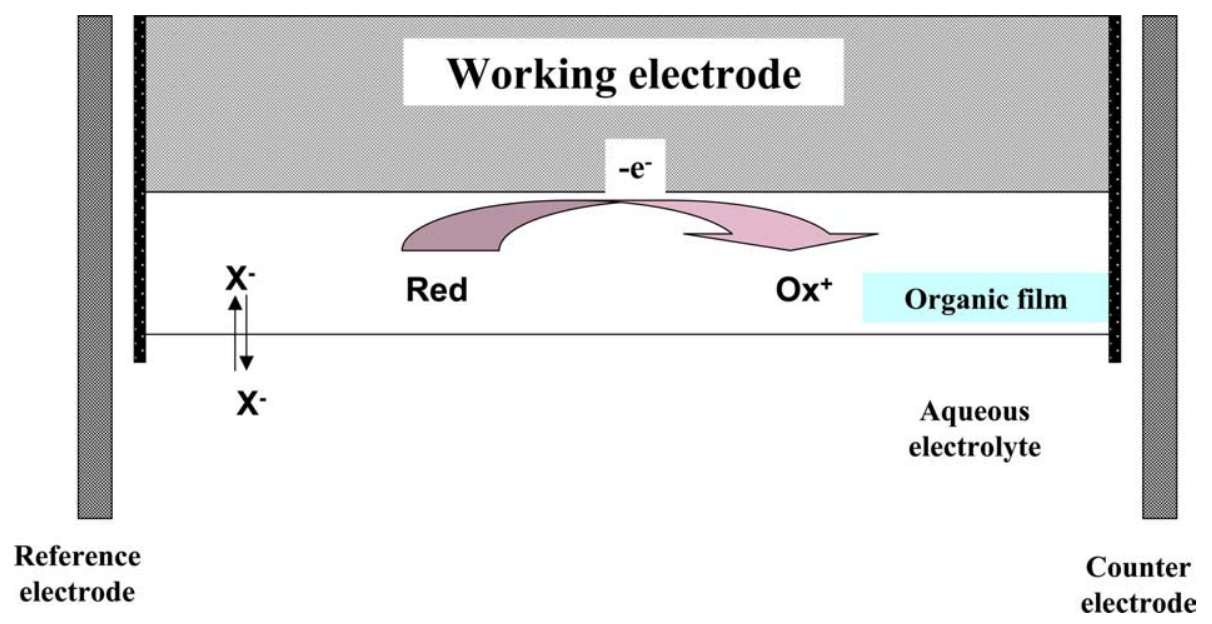

Scheme 1. A solid electrode covered with a micro-film of an organic liquid containing an electroactive probe and immersed in the aqueous electrolyte.

the method is virtually limited to the use of very thin films and to a few types of electrolytes that partition sufficiently between the aqueous and the organic phases.

The recently developed method of Scholz et al. [14,15] (see Scheme 2) consists in a single organic droplet containing an electroactive probe that is attached to the surface of a solid paraffin impregnated graphite electrode and immersed in an aqueous electrolyte. This method provides further simplification of the experimental arrangement and overcomes limitations with respect to the type of aqueous electrolyte. Several recent studies [16-18] demonstrate that a well-defined voltammetric response is obtained without adding any supporting electrolyte to the organic phase regardless of the type of aqueous electrolyte. The unique feature is the presence of a three-phase junction line at which all phases, i.e. the electrode, organic liquid, and the aqueous electrolyte are in intimate contact. Thus, the three-phase junction line provides conditions for electrochemical activity without any constrains. However, it has been already proven that the reaction is not re- stricted exclusively to the three-phase junction line, but it advances to some extend into the organic phase [1921]. For instance, typical cyclic voltammograms of decamethylferrocene $(\mathrm{dmfc})$ oxidation recorded at a low scan rate $(v<100 \mathrm{mV} / \mathrm{s})[14,15]$ exhibit typical properties of an electrode reaction occurring under semi-infinite diffusion conditions. The oxidation of $\mathrm{dmfc}$ to $\mathrm{dmfc}^{+}$in the organic droplet is accompanied by the transfer of counterbalancing anions from the aqueous into the organic phase. Thus, in the course of the voltammetric experiment, both electrochemical processes, i.e., the oxidation of $\mathrm{dmfc}$ to $\mathrm{dmfc}^{+}$and the ion transfer across the $\mathrm{L} \mid \mathrm{L}$ interface, introduce a considerable amount of ions to the organic phase, increasing its conductivity progressively and hence enabling the advancement of the reaction zone from the three-phase junction line into the organic phase. Of course, how far the reaction will advance in the organic phase depends on the time of electrolysis, i.e. the scan rate in cyclovoltammetric experiments. The advancement of the electrochemical reaction zone from the three-phase

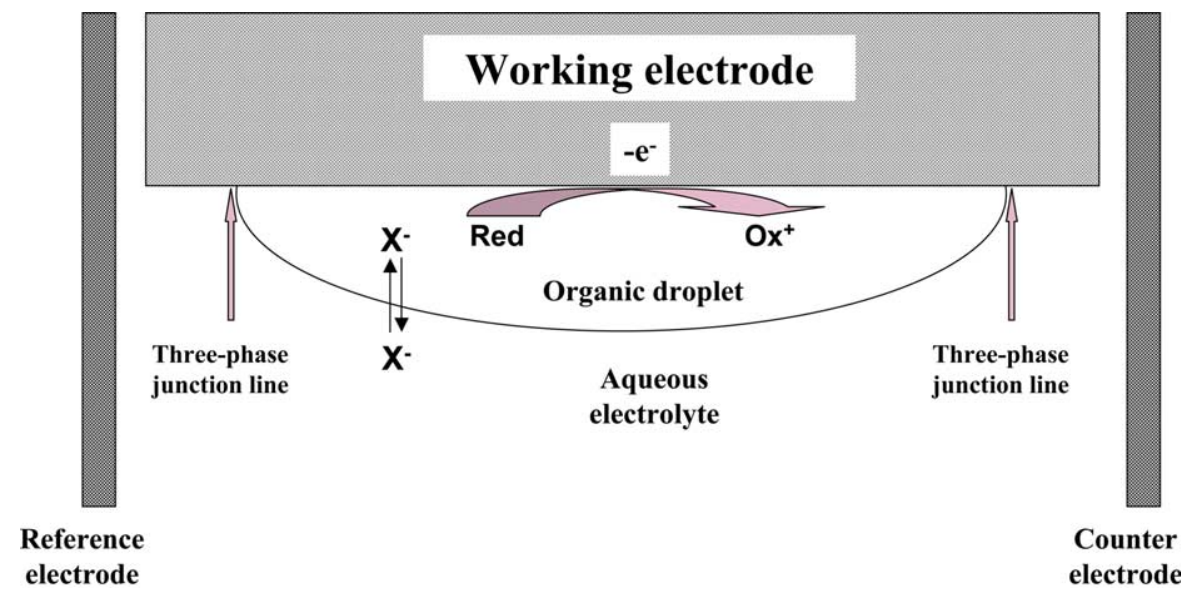

Scheme 2. An organic droplet containing electroactive probe attached to the solid electrode and immersed in an aqueous electrolyte solution. 
boundary line towards the middle of the organic droplet has already been proven by probing the reaction zone by means of a micro-electrode [22].

However, using a fast voltammetric technique such as square-wave voltammetry (SWV), it can be assumed that the reaction is restricted to a "thin film" adjacent to the three-phase junction line. Strictly speaking, this is not a film but a prism lying on a rectangular base. However, for the sake of simplicity, a film can be taken as a model. The time of electrolysis is short to enable considerable advancement of the reaction zone and activation of the organic droplet. The true thickness of the electrically conductive film, in which the reaction can proceed, depends mainly on the partition of the aqueous electrolyte between the aqueous and the organic phases.

Several authors attempted to provide a theoretical basis for the electrode mechanism at the droplet electrode [19-21,23-25]. In the first paper regarding the modelling of this experiment, Myland and Oldham [23] have assumed that the reaction occurs exclusively along the three-phase junction line, which was described geometrically as a micro-hemitoroid. Later on, they have developed a more complex theoretical model considering a thin film of an organic solvent that is free of a supporting electrolyte and sandwiched between the electrode and the aqueous phase [24]. In the theoretical work of Lovric and Scholz [19], the organic droplet was approximated as a conic film, whereas the distribution of electrochemically created ions in the organic phase has been modelled considering a thin film of a constant thickness [25]. Furthermore, Aoki et al. [20,21] have modelled the experiment on the basis of a macroband electrode. It is finally worth noting that Nakatani and Sekine [26,27] investigated also the mass transport of electroactive species at an oil | water interface caused by an electrode reaction. Their electrochemical system consisted of a single micro-oil droplet attached at a micro-electrode in the water phase, but the electrode was not in contact with the electrolyte. The mass transport was modelled as a series combination of the electrode $\mid$ oil interface and the oil $\mid$ water interface. The model is a modification of the Anson film electrode configuration from the viewpoint of mass transport.

Therefore, consistent with the previous authors $[19,24,20,27]$, both the film electrode of Anson and, under certain conditions, the droplet electrode of Scholz can be treated theoretically as a thin-film electrode with a low conductivity of the film, i.e., under conditions of uncompensated resistance. With this motivation, in the present study we analyze theoretically the effect of the uncompensated resistance on the voltammetric response of a simple electrode reaction occurring in a limiting diffusion space. Square-wave voltammetry is used as a working technique. Further the influence of the charge transfer kinetics is also analyzed, keeping in mind the possible similarities between these two phenomena. The theoretical results are compared with experimental results obtained with a single droplet attached to the surface of a graphite electrode, using the reduction of iodine [28], oxidation of $\mathrm{dmfc}[14,15]$, and reduction of azobenzene, all dissolved in nitrobenzene, as model reactions.

\section{Experimental}

All the chemicals used were of analytical purity. 0.1 $\mathrm{mol} / \mathrm{l}$ solutions of decamethylferrocene (ACRÖS, Germany) and iodine (Merck, Germany), as well as a 0.01 $\mathrm{mol} / \mathrm{l}$ solution of azobenzene (Sigma-Aldrich, Germany) were prepared by dissolution of the compounds in water saturated nitrobenzene (NB). A droplet of these solutions with a volume of $1 \mu \mathrm{l}$ was attached to the working paraffin impregnated graphite electrode (PIGE) and immersed into the aqueous electrolyte solutions (saturated with nitrobenzene). Square-wave voltammograms were recorded using the commercial electrochemical measuring system $\mu$ AUTOLAB (Eco-Chemie, Utrecht, Netherlands). $\mathrm{An} \mathrm{Ag} \mid \mathrm{AgCl}$ (saturated $\mathrm{NaCl}$ solution; $E=0.200 \mathrm{~V}$ vs. SHE) was the reference, while the $\mathrm{Pt}$ wire served as an auxiliary electrode. The procedure for preparing the working electrode, i.e. the paraffin impregnated graphite electrode, is described elsewhere [29]. Typical instrumental parameters (if not specified otherwise) were: SW frequency $f=10 \mathrm{~Hz}, \mathrm{SW}$ amplitude $E_{\mathrm{sw}}=50 \mathrm{mV}$, and scan increment $\mathrm{d} E=0.5$ $\mathrm{mV}$. At least three measurements were made for each point presented in Figs. 5-9 and the average values of the peak currents are presented. For all experiments, Millipore Q water was used. All experiments were performed at room temperature.

\section{Mathematical model}

The electrode reaction of two chemically stable species, occurring in a thin film with a thickness $L$, is investigated

$\mathrm{Ox}+n \mathrm{e}^{-} \rightleftarrows$ Red.

For simplicity, the diffusion coefficients of both Ox and Red are assumed to be equal. Furthermore, some effects that under certain conditions could influence the overall electrochemical process at both film and droplet electrodes, such as ion transfer kinetics, membrane potential, Marangoni effects, are not considered in the further mathematical treatment. The following model represents mathematically the electrode reaction:

$\frac{\partial c_{\mathrm{Ox}}}{\partial t}=D \frac{\partial^{2} c_{\mathrm{Ox}}}{\partial x^{2}}$ 
Table 1

List of symbols and abbreviations

\begin{tabular}{|c|c|c|}
\hline Symbol & Meanings of the symbols and abbreviation & Units \\
\hline$\alpha$ & electron transfer coefficient & - \\
\hline$c_{\mathrm{Ox}}$ & concentration of the oxidized form & $\mathrm{mol} \mathrm{cm}^{-3}$ \\
\hline$c_{\text {Red }}$ & concentration of the reduced form & $\mathrm{mol} \mathrm{cm} \mathrm{cm}^{-3}$ \\
\hline$c_{\mathrm{Ox}}^{*}$ & initial concentration of the oxidized form & $\mathrm{mol} \mathrm{cm}^{-3}$ \\
\hline$D$ & diffusion coefficient & $\mathrm{cm}^{2} \mathrm{~s}^{-1}$ \\
\hline $\mathrm{d} E$ & scan increment & $\mathrm{mV}$ \\
\hline$\Delta E_{\mathrm{p} / 2}$ & half-peak width & $\mathrm{mV}$ \\
\hline E & potential & $\mathrm{V}$ \\
\hline$E_{\mathrm{p}}$ & peak potential & $\mathrm{V}$ \\
\hline$E_{\mathrm{Sw}}$ & square-wave amplitude & $\mathrm{mV}$ \\
\hline$E_{\mathrm{Ox} / \mathrm{Red}}^{\emptyset}$ & standard redox potential & $\mathrm{V}$ \\
\hline$f$ & square-wave frequency & $\mathrm{s}^{-1}$ \\
\hline$f_{\max }$ & square-wave frequency associated with the position of the quasireversible maximum & $\mathrm{s}^{-1}$ \\
\hline$F$ & Faraday constant & $\mathrm{C} / \mathrm{mol}$ \\
\hline$\varphi$ & dimensionless potential & - \\
\hline$I$ & current & A \\
\hline$\Delta I_{\mathrm{p}}$ & net-peak current & A \\
\hline$k$ & kinetic parameter & - \\
\hline$k_{\mathrm{s}}$ & standard rate constant & $\mathrm{cm} \mathrm{s}^{-1}$ \\
\hline$L$ & thickness of the film & $\mathrm{cm}$ \\
\hline$\Lambda$ & thickness parameter & - \\
\hline$R$ & gas constant & $\mathrm{J} \mathrm{mol}^{-1} \mathrm{~K}^{-1}$ \\
\hline$R_{\Omega}$ & resistance & $\Omega$ \\
\hline$\rho$ & resistance parameter & - \\
\hline$S$ & electrode surface area & $\mathrm{cm}^{2}$ \\
\hline$t$ & time & s \\
\hline$T$ & thermodynamic temperature & $\mathrm{K}$ \\
\hline$x$ & distance & $\mathrm{cm}$ \\
\hline$\Psi$ & dimensionless current & - \\
\hline$\Delta \Psi_{\mathrm{p}}$ & dimensionless net-peak current & - \\
\hline
\end{tabular}

$\frac{\partial c_{\text {Red }}}{\partial t}=D \frac{\partial^{2} c_{\text {Red }}}{\partial x^{2}}$

$t=0, \quad 0 \leqslant x \leqslant L: \quad c_{\mathrm{Ox}}=c_{\mathrm{Ox}}^{*}, \quad c_{\mathrm{Red}}=0$,

$t>0, \quad x=0: \quad\left(\frac{\partial c_{\text {Ox }}}{\partial x}\right)=-\left(\frac{\partial c_{\text {Red }}}{\partial x}\right)=\frac{I}{n F D S}$,

$t>0, \quad x=L: \quad\left(\frac{\partial c_{\mathrm{Red}}}{\partial x}\right)=\left(\frac{\partial c_{\mathrm{Ox}}}{\partial x}\right)=0$,

$\left(c_{\mathrm{Ox}}\right)_{x=0}=\left(c_{\mathrm{Red}}\right)_{x=0} \exp (\varphi)$,

$\varphi=\frac{n F}{R T}\left(E-E_{\mathrm{Ox} / \mathrm{Red}}^{\emptyset}\right)$.

For the meaning of all symbols and abbreviations see Table 1 . When the resistance is taken into account, the dimensionless potential is defined as $\varphi=(n F / R T)$ $\left(E-E_{\mathrm{Ox} / \text { Red }}^{\emptyset}+I R_{\Omega}\right)$, where $R_{\Omega}$ is the resistance of the film. For the sake of simplicity, in the current model we consider a constant resistance of the film. It should be emphasized that for the film and droplet electrodes, the resistance of the organic phase can vary slightly during the voltammetric experiments due to creation of charged products of the electrode reaction as well as the ingress of counter ions into the organic phase. However, for thin films and short electrolysis time, this effect is not expected to be significant since the conductivity of the film is mainly controlled by the partition of the aqueous electrolyte. For a quasireversible electrode reaction, the following condition holds at the electrode surface:

$\frac{I}{n F S}=k_{\mathrm{s}} \exp (-\alpha \varphi)\left[\left(c_{\mathrm{Ox}}\right)_{x=0}-\left(c_{\mathrm{Red}}\right)_{x=0} \exp (\varphi)\right]$,

where $k_{\mathrm{s}}$ is the heterogeneous standard rate constant in units of $\mathrm{cm} \mathrm{s}^{-1}$. The differential equations (1) and (2) have been solved with the aid of Laplace transforms and the modified step-function method as described in [30]. The solution for a reversible electrode reaction in the form of a recursive formula reads

$$
\Psi_{m}=\frac{1+\frac{\exp \left(\varphi_{m}\right)-1}{50 p \sqrt{f}} \sum_{j=1}^{m-1} \Psi_{j} S_{m-j+1}}{\frac{S_{1}}{50 p \sqrt{f}}\left[\exp \left(\varphi_{m}\right)-1\right]} .
$$

Here the dimensionless current is defined as $\Psi=I$ / $n F S c_{\mathrm{Ox}}^{*} \sqrt{D f}$ and the integration factor $S_{m}$ is $S_{m}=$ $\sum_{i=p(m-1)}^{p m} \Phi_{i}$. The discrete values of the function $\Phi_{i}$ are calculated according to the recursive formula 
$\Phi_{i}=\frac{\frac{1}{\sqrt{\pi i d^{\prime}}}+\frac{\exp \left(\frac{-a^{2}}{4 i d^{\prime}}\right)}{\sqrt{\pi i d^{\prime}}}-\sum_{j=1}^{i-1} \Phi_{i} M_{i-j+1}}{M_{i}-1}$,

where $M_{i}=\operatorname{erfc}\left(a / 2 \sqrt{(i+1) d^{\prime}}\right)-\operatorname{erfc}\left(a / 2 \sqrt{i d^{\prime}}\right)$ and $a=2(L / \sqrt{D})$. In deriving the above formula the main time increment was defined as $d=1 / 50 f$ and the subtime increment $d^{\prime}=d / p$ where $p$ is the number of subintervals, and $f$ is the $\mathrm{SW}$ frequency. In this work the number of subintervals is set to $p=5$.

\section{Theoretical results}

\subsection{Reversible electrode reaction}

The properties of the SW voltammetric response of a reversible electrode reaction occurring in a limiting diffusion space in the absence of uncompensated resistance are mainly controlled by the thickness parameter $\Lambda=L \sqrt{f / D}$, where $L$ is the thickness of the layer, $f$ is the SW frequency and $D$ is the diffusion coefficient. The dimensionless net-peak current $\left(\Delta \Psi_{\mathrm{p}}\right)$ depends sigmoidally on the logarithm of $\Lambda$ (data not shown). Within the interval $-0.2 \leqslant \log (\Lambda) \leqslant 0.1$ the net-peak current increases linearly with $\Lambda$. For $\log (\Lambda)<-0.5$ the diffusion has no effect on the response, and the behavior of the system is similar to a surface confined electrode reaction [31], whereas for $\log (\Lambda)>0.2$, the thickness of the layer

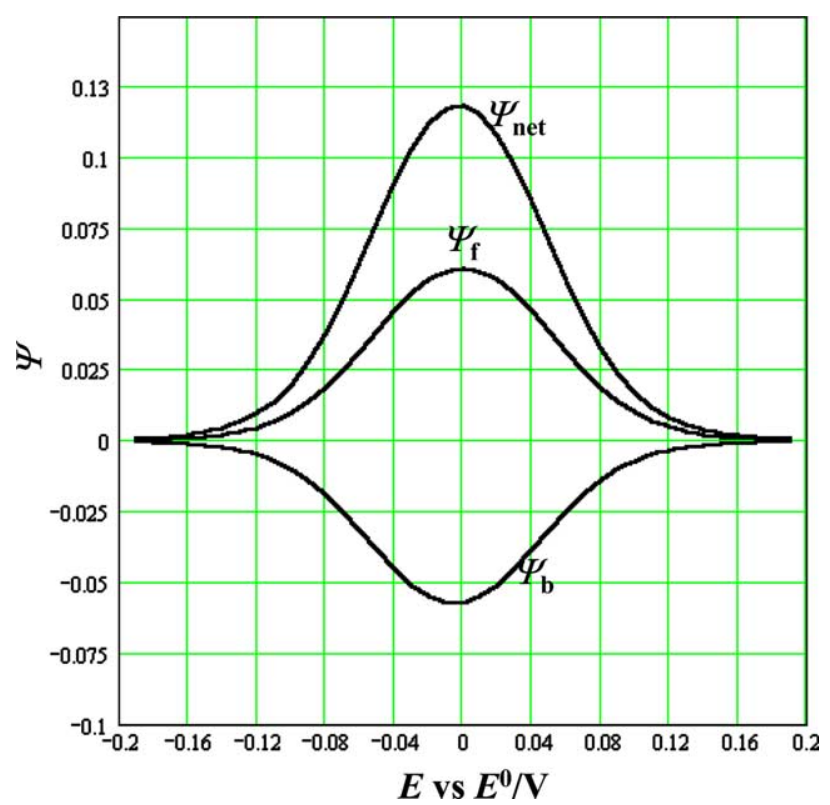

Fig. 1. Dimensionless SW voltammetric response of a reversible electrode reaction occurring in a film with a thickness parameter $\Lambda=0.632$. The signal parameters were: $\mathrm{SW}$ amplitude $n E_{\mathrm{sw}}=50 \mathrm{mV}$ and the scan increment $\mathrm{d} E=10 \mathrm{mV} . \Psi_{\mathrm{f}}, \Psi_{\mathrm{b}}, \Psi_{\text {net }}$ are symbols for forward, backward and net components of the SW voltammetric response, respectively. is bigger than the diffusion layer and the reaction occurs under conditions of semi-infinite planar diffusion.

A typical voltammetric response of a film with a thickness parameter $\Lambda=0.632$ is shown in Fig. 1 . Typical for the film voltammetry is the bell-shape of both the forward and backward components of the SW response. On the contrary, for a semi-infinite diffusion controlled reaction, these components have the shape of a wave. Furthermore, the peak potentials as well as peak currents of both the forward and backward components are virtually identical. The relationship between the real net-peak current and the SW frequency depends on the thickness of the film. For $L>10 \mu \mathrm{m}$ and $D=1 \times 10^{-5} \mathrm{~cm}^{2} \mathrm{~s}^{-1}$, the real net-peak current depends linearly on the square-root of the frequency, over the frequency interval from 10 to 1000 $\mathrm{Hz}$, whereas for $L<2 \mu \mathrm{m}$ the dependence deviates from linearity. For thin films the peak current ratio of the forward and backward components is sensitive to the frequency. For instance, it varies from 1.19 to 1.45 over the frequency interval $10 \leqslant f / \mathrm{Hz} \leqslant 1000$, which is valid for $L<10 \mu \mathrm{m}$ and $D=1 \times 10^{-5} \mathrm{~cm}^{2} \mathrm{~s}^{-1}$. It is important to emphasize that the frequency has no influence upon the peak potential of all components of the response and their values are identical with the formal potential of the redox system.

In the case of an uncompensated resistance, besides the thickness parameter, the reaction is controlled by the dimensionless resistance parameter $\rho=R_{\Omega}\left(n^{2} F^{2} / R T\right)$ $S c_{\mathrm{Ox}}^{*} \sqrt{D f}$, where $R_{\Omega}$ is the resistance of the film. The overall voltammetric behaviour of the system changes with the resistance parameter. The influence of the resistance parameter upon the response depends on the thickness of the layer. Generally, the thicker the film, the smaller the effect of the resistance parameter. The minimal values of the resistance parameter exhibiting an influence on the response are listed in Table 2 for different thicknesses of the film. For $\Lambda<0.949$, all components of the response are appreciably sensitive to the resistance parameter. In Fig. 2 are shown the SW responses of the film with a thickness parameter $\Lambda=0.632$

Table 2

Minimal values of the resistance parameter $\rho_{\min }$ that affect the SW voltammetric response of a reversible electrode reaction for different thicknesses of the film

\begin{tabular}{cl}
\hline$L(\mu \mathrm{m})$ & $\rho_{\min }$ \\
\hline 1 & 0.1 \\
2 & 0.03 \\
5 & 0.04 \\
10 & 2 \\
50 & 3 \\
500 & 4 \\
\hline
\end{tabular}

The conditions of the simulations were: diffusion coefficient $D=1 \times 10^{-5} \mathrm{~cm}^{2} \mathrm{~s}^{-1}$, SW frequency $f=100 \mathrm{~Hz}$, SW amplitude $n E_{\mathrm{sw}}=50 \mathrm{mV}$, and scan increment $\mathrm{d} E=10 \mathrm{mV}$. 

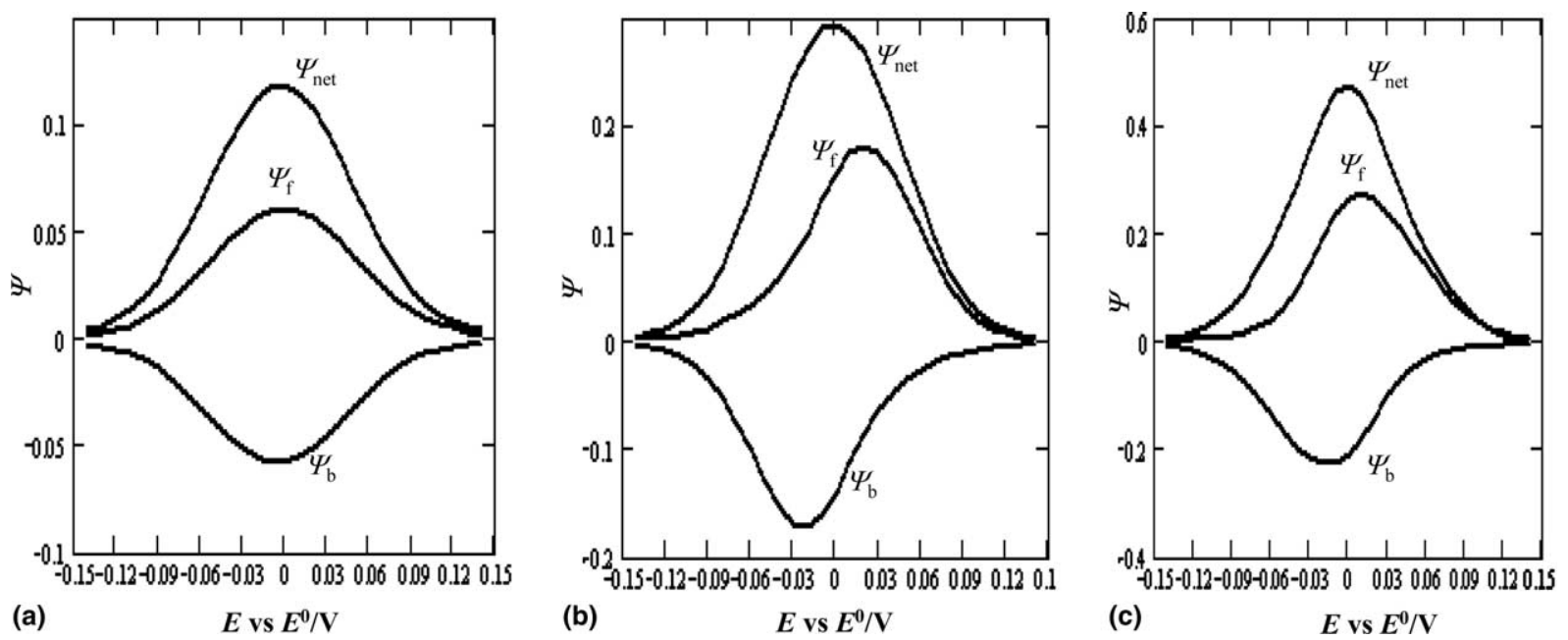

Fig. 2. The effect of the uncompensated resistance on the dimensionless SW voltammograms of a reversible electrode reaction. The resistance parameter was: $\rho=0$ (a), 1 (b) and 3 (c). The other conditions of the simulations were the same as in the caption of Fig. 1.

for three values of the resistance parameter. The increase of $\rho$ displaces the symmetry of the forward and backward components causing the forward (cathodic) branch to shift towards more positive potentials and the backward (anodic) branch towards more negative potentials (see Fig. 2). This remarkable phenomenon originates from specific chronoamperomeric properties of a thin-film electrode reaction. During each potential pulse of the SW voltammetric excitation signal the current diminishes severely with time, as the equilibrium is rapidly established between the species of the reversible couple that diffuse only in a limiting space. Consequently, a low current remains to be measured at the end of each potential pulse. In the case of significant resistance, the redox equilibrium cannot be established so quickly, causing the redox reaction to proceed to a larger extent even at the end of the pulse and thus causing the current to be higher than that without that resistance. For these reasons the maximum of the cathodic component of the response is reached even at more positive, and the maximum of the anodic component at more negative potentials. For comparison, it is interesting to note that for a reaction occurring under semi-infinite diffusion conditions, the influence of the resistance parameter on the positions of the forward and backward components is opposite [32]. Namely, for a reversible semi-infinite diffusion controlled electrode reaction the cathodic components shift towards more negative, and the anodic component towards more positive potential by increasing the resistance parameter. Therefore, this peculiarity can clearly reveal the type of mass transport controlling the voltammetric response. Furthermore, over the interval $0<\rho \leqslant 0.5$ (for $\Lambda=0.632$ ), the difference between the peak potentials of the forward $\left(E_{\mathrm{p}, \mathrm{f}}\right)$ and backward $\left(E_{\mathrm{p}, \mathrm{b}}\right)$ components, $\Delta E_{\mathrm{p}}=E_{\mathrm{p}, \mathrm{f}}-E_{\mathrm{p}, \mathrm{b}}$, increases linearly with $\rho$. For $\rho>5$, a

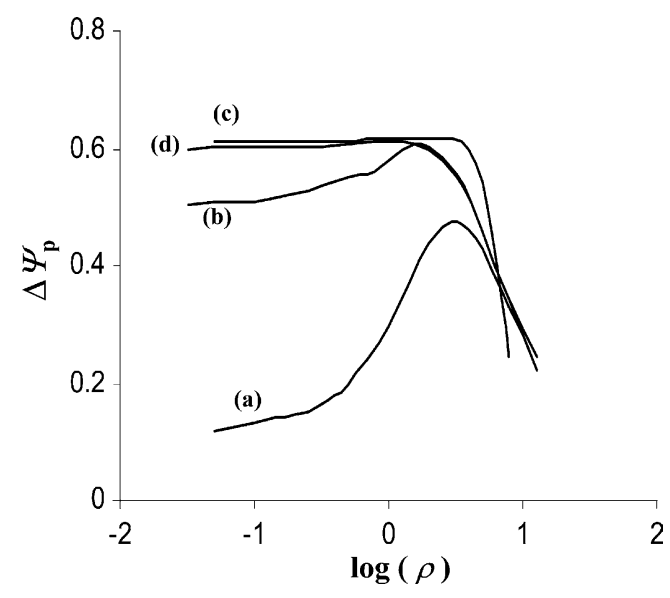

Fig. 3. Influence of the resistance parameter $\rho$ on the dimensionless net-peak current $\Delta \Psi_{\mathrm{p}}$ of a reversible electrode reaction for different thickness parameters. The thickness parameter was: $\Lambda=0.632$ (a), 0.948 (b), 1.581 (c), and 15.811 (d). The other conditions of the simulations were same as in the caption of Fig. 1.

significant distortion in the shape of all components of the response was observed.

The most intriguing effect was observed by studying the dependence of the net-peak current on the resistance parameter. Fig. 3 shows this effect for a series of thickness parameters. Interestingly, for $\Lambda \leqslant 0.949$, the peak current first increases, and after reaching the maximum value it decreases. A parabola can approximate the overall dependence. The maximum of the parabola is associated with a certain critical value of the resistance parameter, $\rho_{\max }$. The critical values of the resistance parameter depend on the thickness parameter obeying the function

$\log \left(\rho_{\max }\right)=-1.2734 \log (\Lambda)+0.2526\left(R^{2}=0.9932\right)$ 
which is valid for $n E_{\mathrm{sw}}=50 \mathrm{mV}$ and $\mathrm{d} E=10 \mathrm{mV}$. The physical meaning of the critical resistance parameter $\rho_{\max }$ is that for a series of electrode reactions occurring under identical conditions, but in films of different conductivities, there is a single reaction exhibiting the highest net SW response. The resistance parameter, ascribed to such reaction, is called the critical resistance parameter.

For a thick layer $(\Lambda>0.949)$ the parabolic dependence between the dimensionless net-peak current and the resistance parameter is practically lost and after being almost constant the peak current decreases monotonically with $\rho$ (see curves c and d in Fig. 3).

In the presence of uncompensated resistance, the frequency exhibits a complex effect upon the response since it affects simultaneously both the thickness parameter $\Lambda$ and the resistance parameter $\rho$. For a film with a thickness of $L \leqslant 2 \mu \mathrm{m}\left(D=1 \times 10^{-5} \mathrm{~cm}^{2} \mathrm{~s}^{-1}\right)$ the real peak current depends sigmoidally on the square root of the frequency, whereas the dimensionless peak current shows a parabolic dependence, with a maximum at the critical frequency $f_{\max }$. Knowing the relation between the critical resistance parameter and the critical frequency, $\rho_{\max }=R_{\Omega}\left(n^{2} F^{2} / R T\right) S c_{\mathrm{Ox}}^{*} \sqrt{D f_{\max }}$, as well as the relation between $\rho_{\max }$ and the thickness parameter $\Lambda$ (see Eq. 5), one can derive the following equation:

$$
\begin{aligned}
\log (L)= & \log \left(\sqrt{\frac{f_{\max }}{D}}\right)+0.198 \\
& -0.785 \log \left(R_{\Omega} \frac{n^{2} F^{2}}{R T} S c_{\mathrm{Ox}}^{*} \sqrt{D f_{\max }}\right) .
\end{aligned}
$$

The latter equation is of particular importance since it enables estimation of the thickness of the layer $L$ by measuring the critical frequency $f_{\max }$, provided the other parameters in Eq. (6) are known.

It is worth noting that in the presence of the uncompensated resistance, the variation of the frequency over the interval $10 \leqslant f / \mathrm{Hz} \leqslant 1000$ caused the peak current ratio of the forward and backward components to increase rapidly from about 1 to 5 , for $L \leqslant 10 \mu \mathrm{m}$, $D=1 \times 10^{-5} \mathrm{~cm}^{2} \mathrm{~s}^{-1}$. Note, that for the same conditions, in the absence of resistance this ratio increases only from 1.19 to 1.45 . Furthermore, the half-peak width also increases monotonically by increasing the resistance parameter. It is however important to stress that though the net-peak current changes dramatically with $\rho$, the net-peak potential is virtually insensitive to the resistance parameter.

\subsection{Quasireversible electrode reaction}

To understand the effect of the uncompensated resistance on a reversible electrode reaction occurring under limiting diffusion conditions, it is instructive to inspect the effect of the charge transfer kinetics, which,

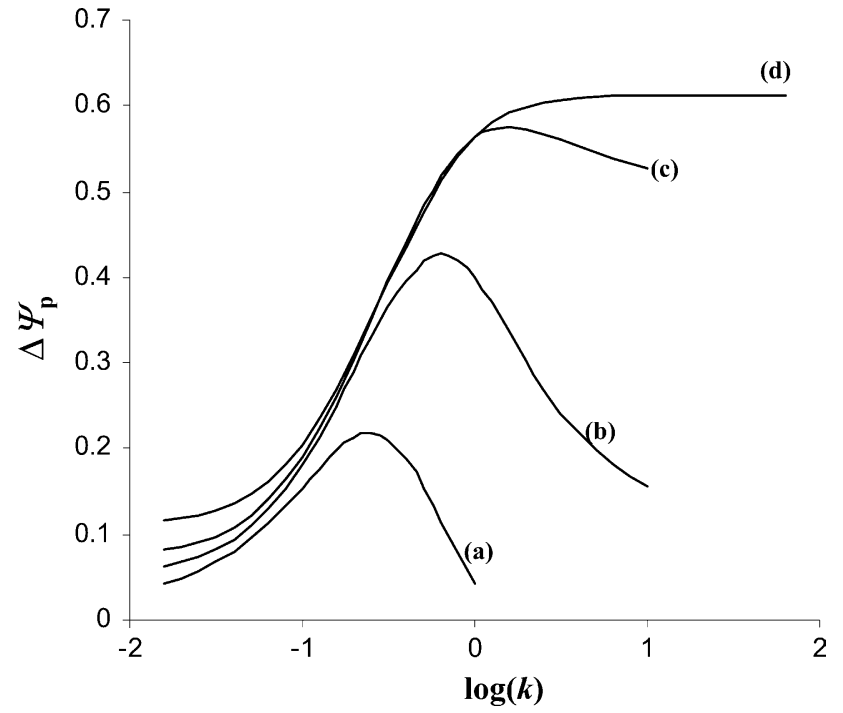

Fig. 4. Influence of the kinetic parameter $k$ on the dimensionless netpeak current $\Delta \Psi_{\mathrm{p}}$ of a quasireversible electrode reaction for different thickness parameters. The thickness parameter was: $\Lambda=0.316$ (a), 0.632 (b), 0.948 (c), and 1.581 (d). The other conditions of the simulations were same as in the caption of Fig. 1.

to the best of our knowledge, has not been studied so far. Besides the thickness parameter $\Lambda$, the response of the quasireversible reaction is controlled by the kinetic parameter $k=k_{\mathrm{s}} / \sqrt{D f}$, where $k_{\mathrm{s}}\left(\mathrm{cm} \mathrm{s}^{-1}\right)$ is the standard rate constant of electron transfer. In Fig. 4 is shown the variation of the dimensionless net-peak current with $\log (k)$ for different thicknesses of the film. For $\Lambda \leqslant 0.949$ a parabolic dependence is observed with the maximum of the parabola located in the quasireversible region. The parabolic dependence of the dimensionless net-peak current on the logarithm of the kinetic parameter is typical for adsorption complicated electrode reactions [31] and for diffusionless surface electrode processes $[33,34]$ and it is known as a "quasireversible maximum". The quasireversible maximum for these types of electrode reactions emerges from the specific chronoamperometric properties due to immobilization of the reactant and the current sampling procedure of square-wave voltammetry. The quasireversible maximum was studied thoroughly in the case of surface electrode reactions and widely exploited for estimation of charge transfer kinetics $[33,35]$. In the present mechanism, the quasireversible maximum is associated with the thin films, when the film is significantly exhausted during the potential scan enabling a repetitive turnover of the electroactive material. The position of the quasireversible maximum depends on the thickness of the film. Thus, the critical kinetic parameter, associated with the maximum $\left(k_{\max }\right)$, obeys the following equation: $\log \left(k_{\max }\right)=1.2649 \Lambda-1$, which is valid for $\alpha=0.5$, $n E_{\mathrm{sw}}=50 \mathrm{mV}$, and $\mathrm{d} E=10 \mathrm{mV}$. Thus, knowing the thickness of the film, and measuring the critical 
frequency associated with the quasireversible maximum, one can estimate the standard rate constant of an electrode reaction with the aid of the following simple relation: $k_{\mathrm{s}}=k_{\max } \sqrt{D f_{\max }}$.

After these considerations, an explanation of the behaviour of a reversible reaction in the presence of an uncompensated resistance can be given. For a reversible reaction, the parabolic dependence between $\Delta \Psi_{\mathrm{p}}$ and $\rho$ was observed for $\Lambda \leqslant 0.949$. On the other hand, the quasireversible maximum exists within the same interval of the thickness parameter, i.e. $\Lambda \leqslant 0.949$. Therefore, the influence of the uncompensated resistance strongly resembles the charge transfer kinetics. The resistance slows down the electrode reaction causing the appearance of the quasireversible maximum even for a reversible electrode reaction. However, due to the strong similarities of both phenomena originating from different sources, it is of particular importance to establish criteria for distinguishing them. The shape of the peak and the half-peak width can serve as simple and safe criteria for this purpose. The uncompensated resistance effect is associated with an increase of the half-peak width, moreover under extreme conditions, by a strong deviation of the bell-shape of the response. On the contrary, under the influence of the charge transfer kinetics the shape of the response and the half-peak width remain virtually unaltered.

\section{Experimental results}

To illustrate qualitatively the theoretically predicted results the three-phase experimental arrangement (see Scheme 2) has been applied. The reduction of iodine and azobenzene, as well as the oxidation of decamethylferrocene, all dissolved in a nitrobenzene droplet, served as model reactions.

The reduction of iodine was studied in the presence of perchloric acid in the aqueous phase. The partition of $\mathrm{HClO}_{4}$ is energetically favored enabling an appreciable electrical conductivity of the region adjacent to the three-phase junction line. A typical SW response of iodine reduction consists of well-developed symmetrical bell-shaped forward (reduction) and backward (oxidation) components (see the inset of Fig. 5). The height of the forward and backward components is almost identical, and their peak potential separation is only $7 \mathrm{mV}$, as is typical for an electrode reaction occurring in a limiting diffusion space (see Fig. 1). The shape of the SW response does not indicate the presence of uncompensated resistance effects.

Fig. 5 shows the variation of the net-peak current of iodine reduction with concentration of aqueous $\mathrm{HClO}_{4}$ solution, for three series of measurements undertaken with SW frequencies of 10,30 and $50 \mathrm{~Hz}$. The net-peak current depends sigmoidally on the $\mathrm{HClO}_{4}$ concentra-

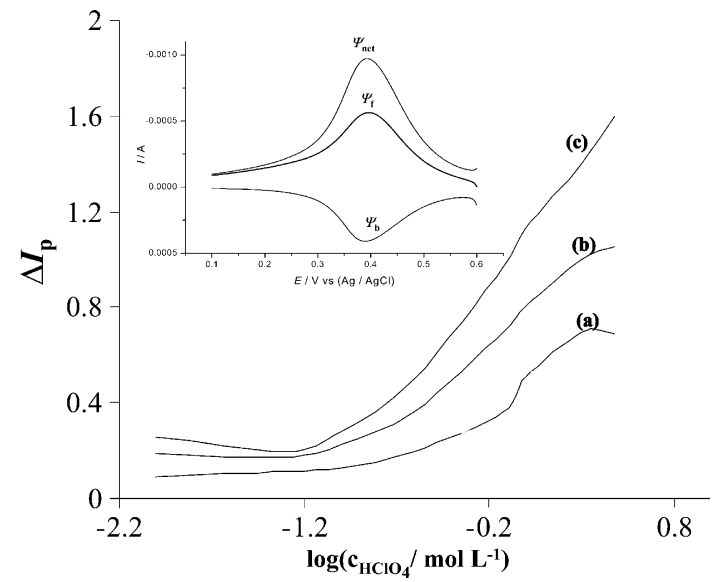

Fig. 5. Dependence of the real net SW peak current $\Delta I_{\mathrm{p}}$ of iodine reduction on the concentration of perchloric acid in the aqueous phase. Besides perchloric acid, the aqueous phase contained $0.1 \mathrm{~mol} / 1 \mathrm{Na}_{2} \mathrm{SO}_{4}$ whereas the organic phase was a $0.1 \mathrm{~mol} / 1$ iodine solution in nitrobenzene. The other experimental conditions were: SW amplitude $E_{\mathrm{sw}}=50 \mathrm{mV}$, scan increment $\mathrm{d} E=0.5 \mathrm{mV}$, and SW frequency $f=10$ (a), 30 (b) and $50 \mathrm{~Hz}$ (c). The inset shows SW voltammograms of iodine reduction recorded at a three-phase arrangement in $2 \mathrm{~mol} / \mathrm{l}$ water solution of $\mathrm{HClO}_{4}$

tion, reaching a plateau for concentrations larger than 2 $\mathrm{mol} / \mathrm{l}$. These dependences resemble the theoretically predicted relationship between the net-peak current and the thickness parameter $\Lambda=L \sqrt{f / D}$ (theoretical data not shown). The larger the concentration of the acid in the aqueous phases the larger is the partitioned concentration of the acid in the organic phase. Hence, the thickness of the electrically conductive film, in which the reaction can occur, increases proportionally by increasing the concentration of the acid in the aqueous phase. The magnitude of the thickness parameter $\Lambda$ can be also varied by the signal frequency. For this purpose, the SW frequency has been altered within the interval from 10 to $300 \mathrm{~Hz}$ in the presence of various acid concentrations in the aqueous phase. For a $2 \mathrm{~mol} / 1 \mathrm{HClO}_{4}$ solution, the net-peak current depends linearly on the square-root of the frequency $\left(R^{2}=0.9916\right)$. This is in agreement with the theoretically predicted behavior for a reversible electrode reaction occurring in the absence of uncompensated resistance. However, in the case of a low conductivity of the film, the increase of the SW frequency will affect simultaneously the thickness parameter $\Lambda=L \sqrt{f / D}$ and the resistance parameter $\rho=R_{\Omega}\left(n^{2} F^{2} / R T\right) S c_{\mathrm{Ox}}^{*} \sqrt{D f}$. To demonstrate this effect a series of experiments have been carried out at lower concentration of the acid. Thus, for $1.00,0.10$, and 0.01 $\mathrm{mol} / \mathrm{l}$ of $\mathrm{HClO}_{4}$ in the aqueous phase, the squares of the coefficients of the linear regression line of the dependence $\Delta I_{\mathrm{p}}$ vs $f^{1 / 2} \Delta I_{\mathrm{p}}$ is the real net-peak current) are $0.9903,0.9525$, and 0.9317 , respectively. Obviously, deviations from linearity occur, indicating that under these conditions the influence of the resistance effect becomes detectable. 


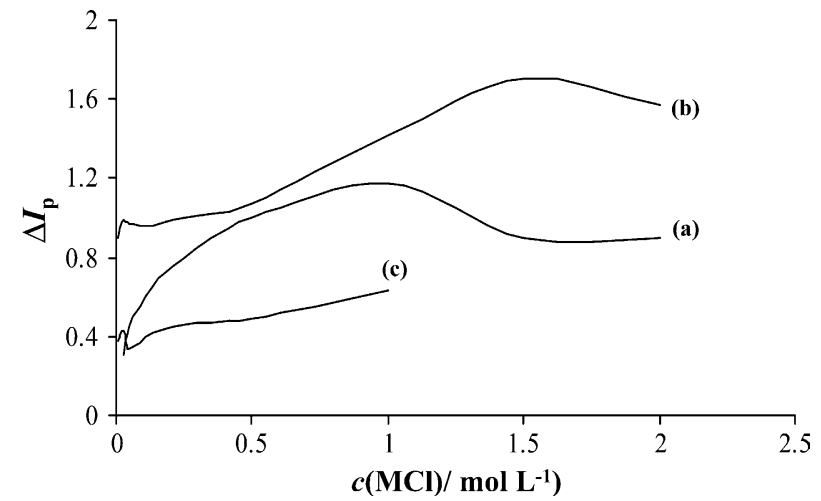

Fig. 6. Dependence of the real net SW peak current of dmfc oxidation on the concentration of $\mathrm{NaCl}$ (a) tetramethylammonim chloride (b), and tetrabutylammonium chloride (c). The symbol $\mathrm{MCl}$ used in the abscissa title is a common abbreviation for all three salts. Besides the respective salt, the aqueous phase contained $0.1 \mathrm{~mol} / 1 \mathrm{Na}_{2} \mathrm{SO}_{4}$. The organic phase was a $0.1 \mathrm{~mol} / 1 \mathrm{dmfc}$ solution in nitrobenzene. The other experimental conditions were: SW frequency $f=20 \mathrm{~Hz}, \mathrm{SW}$ amplitude $E_{\mathrm{sw}}=50 \mathrm{mV}$, and scan increment $\mathrm{d} E=0.5 \mathrm{mV}$.

The influence of uncompensated resistance is more pronounced in the case of $\mathrm{dmfc}$ dissolved in nitrobenzene and immersed in the aqueous phase containing $\mathrm{Na}_{2} \mathrm{SO}_{4}$ as an electrolyte. The partition of $\mathrm{Na}_{2} \mathrm{SO}_{4}$ is energetically unfavourable, restricting the occurrence of reaction to a very thin film with a low conductivity that is adjacent to the three-phase junction line. The electrical conductivity of the film can be partially controlled by adding an additional electrolyte to the aqueous phase,

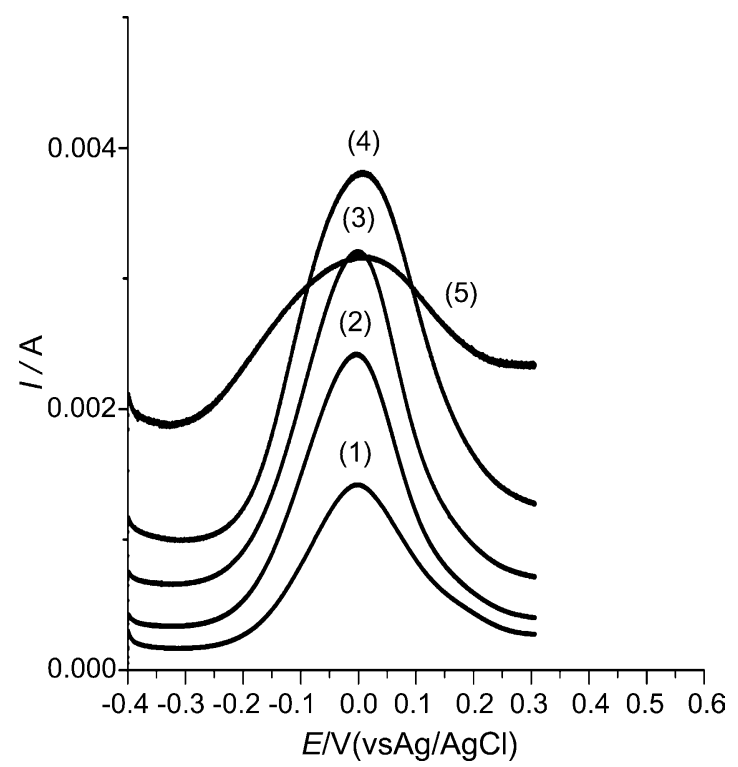

Fig. 7. The effect of the frequency on the SW voltammograms for oxidation of dmfc dissolved in nitrobenzene at a concentration of 0.1 $\mathrm{mol} / \mathrm{l}$. The aqueous phase contained a $0.5 \mathrm{~mol} / \mathrm{l}$ solution of $\mathrm{NaNO}_{3}$. The SW frequency was: 50 (1), 100 (2), 200 (3), 500 (4), and $1000 \mathrm{~Hz}$ (5). The other experimental conditions were: $\mathrm{SW}$ amplitude $E_{\mathrm{sw}}=50$ $\mathrm{mV}$ and scan increment $\mathrm{d} E=0.1 \mathrm{mV}$. which can be transferred more easily into the organic phase than $\mathrm{Na}_{2} \mathrm{SO}_{4}$. Fig. 6 shows the dependence of the net-peak current on the concentration of $\mathrm{NaCl}$, tetramethylammonim chloride (TMACl), and tetrabutylammonium chloride (TBACl) present in the aqueous phase in addition to the $0.1 \mathrm{~mol} / 1$ concentration of $\mathrm{Na}_{2} \mathrm{SO}_{4}$. This analysis corresponds to the variation of the resistance parameter $\rho$ of the system, by varying the resistance of the film. Interestingly, non-linear dependences associated with maxima were observed in the case of $\mathrm{NaCl}$ and TMACl, which is in accordance with the theoretical data presented in Fig. 3.

As previously mentioned, the idea to vary the resistance parameter can be also achieved by adjusting the signal frequency $\left(\rho=R_{\Omega}\left(n^{2} F^{2} / R T\right) S c_{\mathrm{Ox}}^{*} \sqrt{D f}\right)$. Fig. 7 shows the influence of the frequency on the SW response of $\mathrm{dmfc}$. The height of the response is not proportional to the SW frequency. The response increases up to a frequency of $500 \mathrm{~Hz}$; however above this value the height of the peak diminishes. At the same time the halfpeak width increases permanently. At the frequency of

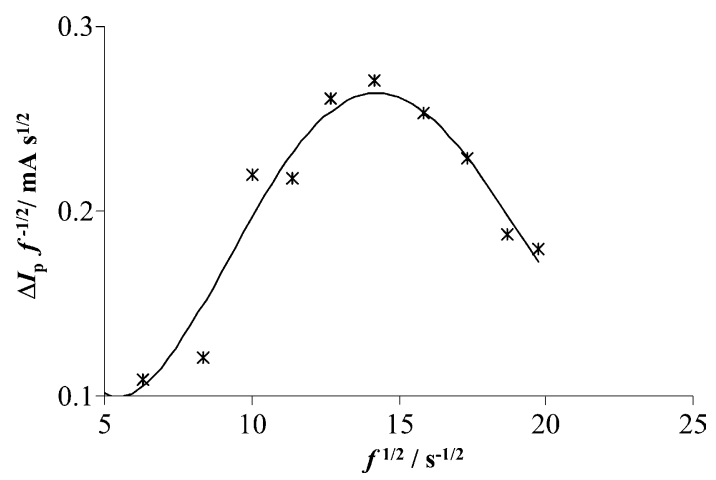

Fig. 8. The influence of the SW frequency on the ratio of the SW peak currents and the square root of the frequency for oxidation of dmfc. The aqueous phase contained $1 \mathrm{~mol} / 1 \mathrm{LiNO}_{3}$ solution. The other conditions were same as in the caption of Fig. 6 .

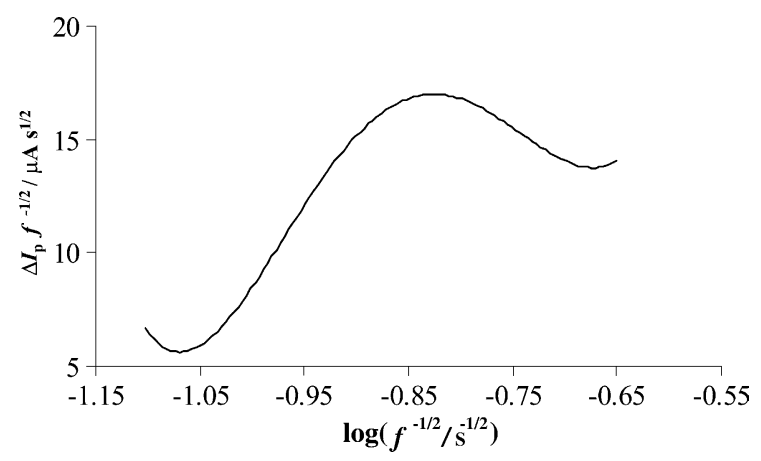

Fig. 9. Quasireversible maximum of azobenzene. The organic phase contained $0.01 \mathrm{~mol} / \mathrm{l}$ azobenzene dissolved in nitrobenzene, whereas the aqueous phase contained $1 \mathrm{~mol} / \mathrm{HNO}_{3}$. The other experimental conditions were: SW amplitude $E_{\mathrm{sw}}=100 \mathrm{mV}$, and scan increment $\mathrm{d} E=1 \mathrm{mV}$. 
$1000 \mathrm{~Hz}$ the response deviates strongly from the bell shape (see curve 5 in Fig. 7). In contrast to the behavior of the peak height and half-peak width, the peak potential remained unchanged and insensitive to the influence of the resistance of the system. All these features of the response fit well with the theoretically predicted behavior in the presence of uncompensated resistance. Moreover, Fig. 8 shows the dependence of the ratio $\Delta I_{\mathrm{p}} f^{-1 / 2}$ on $f^{1 / 2}$ for the oxidation of dmfc. Note that the ratio $\Delta I_{\mathrm{p}} f^{-1 / 2}$ corresponds to the dimensionless netpeak current and the $f^{1 / 2}$ to the resistance parameter $\rho$. The dependence $\Delta I_{\mathrm{p}} f^{-1 / 2}$ vs $f^{1 / 2}$ is associated with a well-developed maximum. The data presented in both Figs. 6 and 8 confirm the theoretically predicted parabolic dependence of the net-peak current on the resistance parameter for a reversible electrode reaction in thin-film voltammetry (see Fig. 3).

Finally, the reduction of azobenzene in a nitrobenzene droplet was studied as a model for a quasireversible electrode reaction occurring under limiting diffusion conditions. The reaction was studied in the presence of 1 mol/l aqueous $\mathrm{HNO}_{3}$ solution, since the two-electron reduction of azobenzene is accompanied by the addition of two protons to form hydrazobenzene. Fig. 9 shows the relationship between the ratio $\Delta I_{\mathrm{p}} f^{-1 / 2}$ and the logarithm of the inverse square-root of the frequency. Again, a parabolic dependence was observed. It is, however, important to note that the half-peak width did not vary by increasing the frequency, which is opposite to the behavior of the system under the influence of an uncompensated resistance, as demonstrated in the case of dmfc. Therefore, the parabolic relationship depicted in Fig. 9 originates mainly from kinetic effects, presenting the quasireversible maximum of azobenzene electrode reaction occurring in a thin-film. To the best of our knowledge it is the first experimental demonstration of the quasireversible maximum in SW thin-film voltammetry.

\section{Acknowledgements}

V.M. thanks A.V. Humboldt-Stiftung for providing a Return Research Fellowship; R.G. thanks Deutscher Akademische Austauschdienst (DAAD) for provision of a Ph.D. scholarship, and F.S. acknowledges support by Deutsche Forschungemeinschaft (DFG) and Fonds der Chemischen Industrie (FCI).

\section{References}

[1] H.H. Girault, D.J. Schiffrin, in: A.J. Bard (Ed.), Electroanalytical Chemistry, vol. 15, Marcel Dekker, New York, 1989.

[2] T. Osakai, K. Ebina, in: A.G. Volkov (Ed.), Liquid Interfaces in Chemical, Biological, and Pharmaceutical Applications, Marcel Dekker, New York, 2001, pp. 23-49.

[3] S. Wilke, H. Wang, J. Electroanal. Chem. 475 (1999) 9.

[4] S. Wilke, T. Zerihun, J. Electroanal. Chem. 515 (2002) 52.

[5] S. Wilke, T. Zerihun, Electrochim. Acta 44 (1998) 15.

[6] S. Wilke, H. Wang, H.H. Girault, J. Electroanal. Chem. 436 (1997) 53.

[7] F. Reymond, G. Lagger, P.-A. Carrupt, H.H. Girault, J. Electroanal. Chem. 451 (1998) 59.

[8] C. Shi, C. Anson, Anal. Chem. 70 (1998) 3114

[9] C. Shi, C. Anson, J. Phys. Chem. B. 102 (1998) 9850.

[10] C. Shi, F.C. Anson, J. Phys. Chem. B 103 (1999) 6283.

[11] T.D. Chung, F.C. Anson, Anal. Chem. 73 (2001) 337.

[12] C. Shi, C. Anson, J. Phys. Chem. B 105 (2001) 1047.

[13] C. Shi, C. Anson, J. Phys. Chem. B 105 (2001) 8963.

[14] F. Scholz, Š. Komorsky-Lovrić, M. Lovrić, Electrochem. Commun. 2 (2000) 112.

[15] Š. Komorsky-Lovrić, M. Lovrić, F. Scholz, J. Electroanal. Chem. 508 (2001) 129

[16] Š. Komorsky-Lovrić, K. Riedl, R. Gulaboski, V. Mirčeski, F. Scholz, Langmuir 18 (2002) 8000;

Š. Komorsky-Lovrić, K. Riedl, R. Gulaboski, V. Mirčeski, F. Scholz, Langmuir 19 (2003) 3090.

[17] R. Gulaboski, V. Mirčeski, F. Scholz, Electrochem. Commun. 4 (2002) 277

[18] R. Gulaboski, K. Riedl, F. Scholz, Phys. Chem. Chem. Phys. 5 (2003) 1284.

[19] M. Lovrić, F. Scholz, J. Electroanal. Chem. 540 (2003) 89.

[20] P. Tasakorn, J. Chen, K. Aoki, J. Electroanal. Chem. 533 (2002) 119.

[21] K. Aoki, P. Tasakorn, J. Chen, J. Electroanal. Chem. 542 (2003) 51.

[22] M. Donten, Z. Stojek, F. Scholz, Electrochem. Commun. 4 (2002) 324.

[23] J.C. Myland, K.B. Oldham, Electrochem. Commun. 2 (2000) 541.

[24] J.C. Myland, K.B. Oldham, J. Electroanal. Chem. 530 (2002) 1.

[25] M. Lovrić, Š. Komorsky-Lovrić, Electrochem. Commun. 5 (2003) 637.

[26] K. Nakatani, T. Sekine, J. Colloid Interface Sci. 225 (2000) 251.

[27] K. Nakatani, T. Sekine, Langmuir 16 (2000) 9256.

[28] V. Mirčeski, F. Scholz, J. Electroanal. Chem. 522 (2002) 189.

[29] F. Scholz, B. Meyer, in: A.J. Bard, I. Rubinstein (Eds.), Electroanalytical Chemistry. A Series of Advances., vol. 20, Marcel Dekker, New York, 1998, p. 1.

[30] V. Mirčeski, J. Electroanal. Chem. 545 (2003) 29.

[31] M. Lovrić, in: F. Scholz (Ed.), Electroanalytical Methods, Guide to Experiments and Applications, Springer, Berlin, Heidelberg, 2002, p. 111.

[32] V. Mirčeski, M. Lovrić, J. Electroanal. Chem. 497 (2001) 114.

[33] Š. Komorsky-Lovrić, M. Lovrić, Anal. Chim. Acta 305 (1995) 248.

[34] V. Mirčeski, M. Lovrić, Electroanalysis 9 (1997) 1283.

[35] V. Mirčeski, M. Lovrić, B. Jordanoski, Electroanalysis 11 (1999) 660. 